\title{
Manipulating the FLS 'proteoglycan switch' could offer a new approach to RA therapy
}

$\mathrm{T}$

he invasiveness and migration of fibroblast-like synoviocytes (FLS) in arthritis is regulated by a phosphatase-dependent 'proteoglycan switch', and pharmacological manipulation of this mechanism could offer a way to directly target these important effector cells, according to new research reported in Science Translational Medicine. The study by Doody et al. suggests that drugs targeting receptor-type tyrosine-protein phosphatase sigma (RPTP $\sigma$, encoded by PTPRS) might provide a novel approach to the treatment of rheumatoid arthritis (RA).

"RPTP $\sigma$ is a transmembrane enzyme known to bind proteoglycans through its extracellular domain, and to regulate axonal elongation in response to cell surface and extracellular proteoglycans in neurons," explains the study's lead author, Nunzio Bottini. "Since the joint and synovial fluid are enriched with proteoglycans, we thought RPTP $\sigma$ was likely to play a key role in the regulation of signalling in FLS as well. The fact that RPTP $\sigma$ is believed to be expressed at [a] low level or not at all in immune cells made it an even better candidate for studies in FLS."

The team of researchers demonstrated that expression of Ptprs is induced in FLS during arthritis progression in mice with $\mathrm{K} / \mathrm{B} \times \mathrm{N}$ serum-induced arthritisa model of RA in which FLS have a strong pathogenic role. Moreover, treatment of mouse and human FLS with exogenous chondroitin sulfate proteoglycans (CSPGs) inhibited the serum-induced migration and invasiveness of the cells in an RPTP $\sigma$-dependent manner.

They next tested the effect of a recombinant RPTP $\sigma$ protein on FLS in vitro and in vivo. "We hypothesized that the recombinant protein would act as a decoy, able to detach endogenous RPTP $\sigma$ from proteoglycans on the surface of FLS," Bottini recounts. "Our theory was that by detaching the RPTP $\sigma$ from proteoglycans we would achieve activation of the phosphatase with consequent inhibition of pathogenic FLS intracellular signalling." Indeed, the decoy protein reduced the invasiveness, migratory capacity and attachment to cartilage of FLS, and further experiments strongly suggested that both the decoy protein and RPTPo interact mainly with the HSPG syndecan- 4 on the cell surface.

Turning to the intracellular mechanisms controlled by the proteoglycan switch, Doody et al. showed that the effects of the decoy protein on FLS migration are mediated by tyrosine dephosphorylation and disrupt cytoskeletal localization of ezrin, a substrate of RPTPo. Ezrin is a cytoskeleton-associated protein, the phosphorylation of which is required for the motility and thus invasiveness of FLS in RA.

Together, the findings support a model of the proteoglycan switch whereby binding of RPTP $\sigma$ with syndecan- 4 on the surface of FLS maintains the phosphatase in an inactive state. Treatment of FLS with the RPTP $\sigma$ decoy protein disrupts this inhibitory effect and activates RPTP $\sigma$, which, in turn, dephosphorylates ezrin. "This is a novel and intriguing concept that strengthens the role of cell-proteoglycan interactions in the transformation of FLS in arthritis," enthuses Thomas Pap of University Hospital Münster, who was not involved in the study.

The in vivo therapeutic efficacy of the decoy protein correlated with its ex vivo effects on FLS. "Injections of the protein reversed arthritis in the $\mathrm{K} / \mathrm{B} \times \mathrm{N}$ mouse model and prevented cartilage invasion by co-implanted human RA FLS," says Bottini.

"Together, the data suggest that RPTP $\sigma$ is a candidate target for anti-RA therapies," asserts Bottini. He continues, "The mechanism of action of anti-RPTP $\sigma$ therapies is likely to be different from

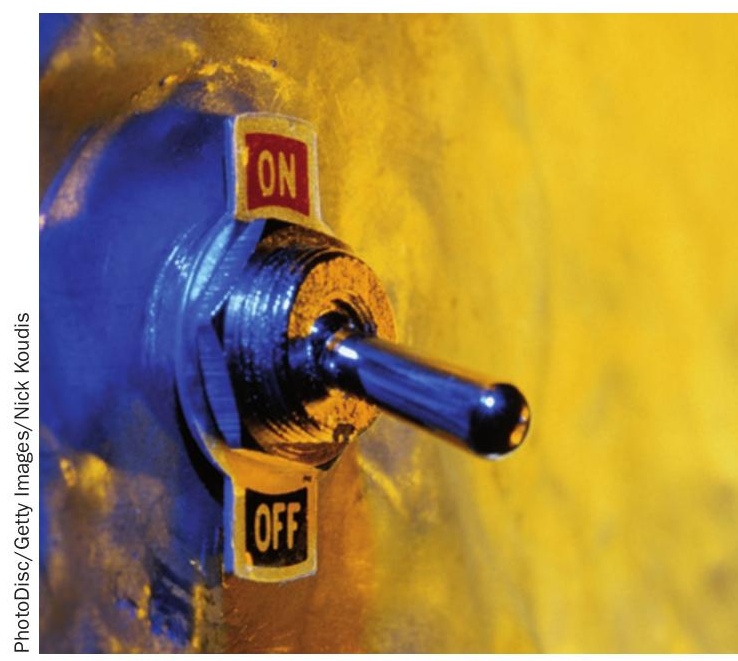

[those of] all other known anti-RA agents." Therapeutic agents that target the invasiveness and proinflammatory action of FLS without suppressing the function of immune cells could be used as adjunctive therapies in patients who are unable to achieve remission with current immunetargeted therapies, or might even be used as monotherapy in those patients with RA whose disease is driven primarily by FLS.

"This is an excellent and very interesting study that may help explain some poorly understood phenomena in arthritis pathology and that certainly has the potential of leading to novel therapeutic strategies for the disease," Pap concludes. "The next steps should be to determine the relevance of these findings for human disease entities, particularly for RA as compared to other conditions, and the identification of the exact nature and the mechanisms by which RPTP $\sigma$ and syndecan- 4 interact."

Sarah Onuora 